\title{
Performance of the ETSI SmartBAN system in the interfered IEEE 802.15.6 channel
}

\author{
Harri Viittala, Matti Hämäläinen \\ Centre for Wireless Communications \\ University of Oulu \\ Oulu, Finland \\ \{harri.viittala, matti.hamalainen\}@oulu.fi
}

\author{
Lorenzo Mucchi \\ Dept. of Information Engineering \\ University of Firenze \\ Firenze, Italy \\ lorenzo.mucchi@unifi.it
}

\begin{abstract}
The ETSI TC SmartBAN defined system is designed for body area networks supporting both on-body links and links to implanted devices. The system operates at the $2.4 \mathrm{GHz}$ ISM band, where are also other wireless radio systems, such as Bluetooth and WiFi. The SmartBAN system simulation model has been created to Matlab and the system performance was studied in an interfered IEEE 802.15.6 channel model 3. The interference model is based on the measurement campaigns carried out in the Oulu university hospital. By using these simulation models, a comparative analysis between $B C H$ encoding schemes $(127,113)$ and $(36,22)$ on the system performance was carried out. It is shown that higher coding rate has a better performance in the presence of interference but it needs a repetition method to gain an acceptable frame error rate.
\end{abstract}

\section{Keywords-BCH, PHY, SmartBAN.}

\section{INTRODUCTION}

The ETSI Smart Body Area Network (SmartBAN) is a technical committee (TC) established in 2013 to define and specify European standards for low power physical layer (PHY) [1], medium access control (MAC) layer [5] and light data presentation formats for smart body area networks [3]. In addition, ETSI TC SmartBAN is studying corresponding radio environment for coexistence reasons. SmartBAN is a body area network supporting on-body links and links to implanted devices and a network is organized around a hub (coordinator) following a star topology. SmartBAN introduces smartness for control, network management, heterogeneity and interoperability. BAN may include nodes from different manufacturers, having dissimilar processing functionalities or not requiring alike resources. Therefore, heterogeneity in terms of node models and profiles, data gathered, communication protocols and applications is presented. Smartness in heterogeneous management is assigned by introducing common semantic approach, i.e., having an open data model dedicated to SmartBAN including conflict resolution and similarity detection. Another smart feature will be coexistence management done by a coordinator. It could follow cognitive approach by implementing mechanism to sense a channel and switch to less occupied frequency band. [4]

SmartBAN operates within the frequency band 2401-2481 $\mathrm{MHz}$, where channels are arranged in the blocks of $2 \mathrm{MHz}$ [1]. Standardized radio technologies (such as Bluetooth and WiFi) and proprietary devices share the same $2.4 \mathrm{GHz}$ ISM band. Medical devices and monitoring systems require high reliability communication links and interference may lead to significant performance degradation. Therefore, it is essential to study how interference affects the SmartBAN system performance.

ETSI TC SmartBAN formed a specialist task force (STF) to build the first simulation model based on the SmartBAN communication system. This STF team was known as STF511: SmartBAN performance and coexistence verification $(P C V)$. The main objective of the STF was to verify the performance of the system using computer simulations. The results provided by the STF indicated that SmartBAN needs more efficient error control and correction mechanisms to mitigate interference coming from other wireless systems. In this paper, it is studied if the shortened code from the primitive $\mathrm{BCH}(127,113)$ code used in the header encoding improves the SmartBAN system performance.

The paper is organized as follows. Chapter II discusses the simulator model including the applied channel model, interference scenarios and coding schemes. Simulation results are given and discussed in Chapter III. The paper is concluded in Chapter IV.

\section{SMARTBAN COMMUNICATION SYStem SimUlator}

To study and evaluate the performance of the SmartBAN system, the software simulator using Matlab with Simulink was developed. The details of the simulator are given in [5]. The simulator was modified to study the shortened version of the $\mathrm{BCH}(127,113)$ code, and is discussed in Section II/B in details.

Fig. 1 depicts the PHY layer block diagram of the developed simulator. The MAC protocol data unit (MPDU) is encoded using the $\mathrm{BCH}$ encoder followed by insertion of headers and preamble. Data is modulated with Gaussian frequency shift keying (GFSK) with the modulation index $(h)$ of 0.5 and bandwidthtime product $(B T)$ of 0.5 . The PHY layer protocol data unit (PPDU) is repeated as introduced in the next section.

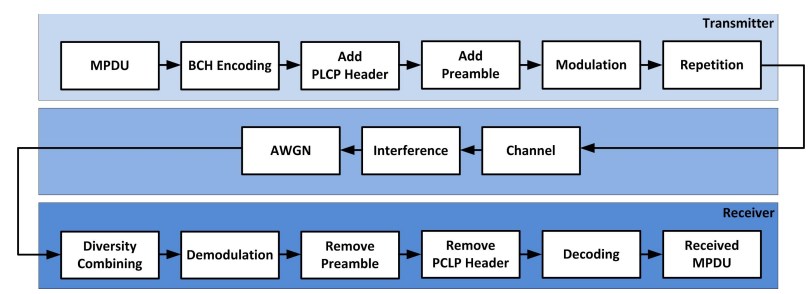

Fig. 1. Schematic of the simulator. 
The transmitted signal goes through a fading channel, which is assumed constant for each repeated PPDU. After that, interference and noise are added to the desired signal. The applied channel model is the IEEE 802.15 .6 body surface to body surface CM3 (Scenario S4 \& S5) for $2.4 \mathrm{GHz}$ [6], where flat smallscale fading is represented by a Ricean distribution with $K_{\mathrm{dB}}$ factor

$$
K_{\mathrm{dB}}=K_{0}-m_{\mathrm{K}} P L_{\mathrm{dB}}+\sigma_{\mathrm{K}} n_{\mathrm{K}},
$$

where $K_{0}$ is $30.6 \mathrm{~dB}, m_{\mathrm{K}}$ is $0.43 \mathrm{~dB} / \mathrm{cm}, \sigma_{\mathrm{K}}$ is $3.4 \mathrm{~dB}$ and $n_{\mathrm{K}}$ is Gaussian random variable with zero mean and unit variance. Pathloss $\left(P L_{\mathrm{dB}}\right)$ is given by

$$
P L_{\mathrm{dB}}=-10 \log _{10}\left(P_{0} e^{-m_{0} d}+P_{1}\right)+\sigma_{\mathrm{P}} n_{\mathrm{P}}[\mathrm{dB}]
$$

where $P_{0}=-25.8 \mathrm{~dB}, m_{0}=2.0 \mathrm{~dB} / \mathrm{cm}, P_{1}=-71.3 \mathrm{~dB}, \sigma_{\mathrm{P}}=3.6$ $\mathrm{dB}$ and $d$ is the distance. After passing the fading channel, an inband interference and the additive white Gaussian noise (AWGN) are added to a signal. The interference is modeled as presented in Section II/B.

The received PPDUs are combined by using the equal gain combining (EGC) method. The demodulator applies a correlator followed by a maximum-likelihood sequence detector (MLSD). After demodulation, preamble and header are removed and data is decoded.

\section{A. Channel coding}

The SmartBAN PHY technical specification defines repetition and forward error control (FEC) coding to reduce and correct errors. The simple repeat code to transmit the entire PPDU two or four times can be used. After repetition, the repeated PPDUs are treated as one single PPDU [1]. This is shown in Fig. 2.

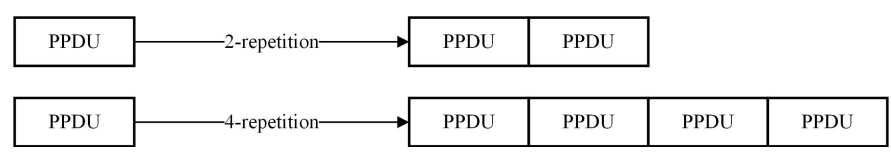

Fig. 2. 2- and 4- time repetition coding.

For error control coding, SmartBAN may apply a systematic $\operatorname{BCH}(n, k)$ code, where $n=127$ and $k=113$. The generator polynomial $g(x)$ of the code is

$$
g(x)=x^{14}+x^{9}+x^{8}+x^{6}+x^{4}+x^{2}+x+1 .
$$

The encoding process is depicted in Fig. 4, and it starts by calculating the number of padding bits into a medium access protocol data unit (MPDU) as

$$
N_{\text {padding }}=\left\lceil\frac{L_{M P D U}}{k}\right\rceil * k-L_{M P D U}
$$

where $L_{\mathrm{MPDU}}$ is the length of the MPDU and $k$ is the length of the message word, i.e., 113 in this case. Next step is to partition the padded MPDU into subpackets with length of $k$. After portioning, parity bits are computed and padded zero bits from the last $m$ subpacket are removed. Finally, the parity bits are appended to each subpacket and the physical layer service data unit (PSDU) is assembled.

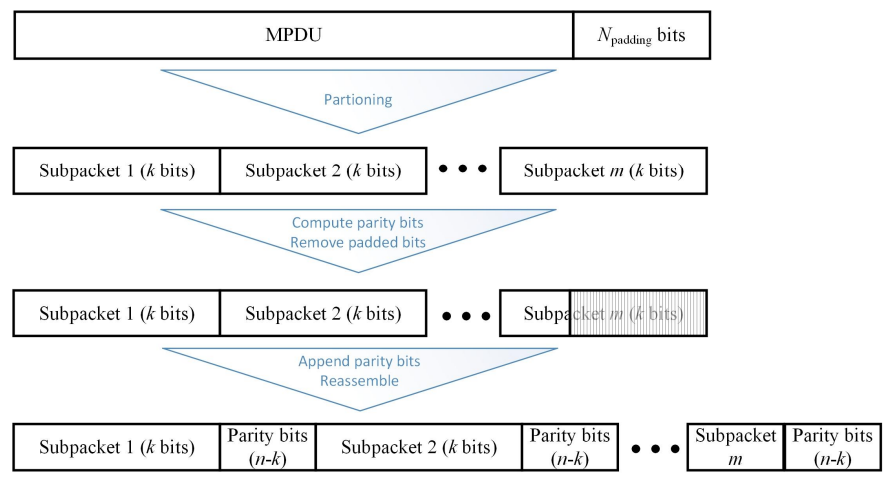

Fig. 3. $\mathrm{BCH}(127,113)$ encoding process.

The shortened $\operatorname{BCH}(36,22)$, derived from the $\mathrm{BCH}(127,113)$, shall be employed for the physical layer convergence protocol (PLCP) header. In this paper, we apply this coding rate also for PSDU instead of $\mathrm{BCH}(127,113)$ as defined in [1]. The encoding process differs slightly from the process discussed above, and it is depicted in Fig. 4. Now, the subpacket length $\left(k_{\mathrm{S}}\right)$ is 22 bits and $N_{\text {padding }}$ bits are calculated, as defined in Eq. (4) and using $k=k_{\mathrm{S}}$. The padded MPDU is partitioned into subpackets having length of 22 bits, and 91 zero bits are appended for each subpacket. The parity bits are computed by using the $\mathrm{BCH}(127,113)$ generator polynomial for each subpacket. Sequentially, padded 91 zero bits are removed from subpackets, and $N_{\text {padding }}$ bits from the last subpacket. Finally, the PSDU is set up by assembling the subpackets including generated parity bits.

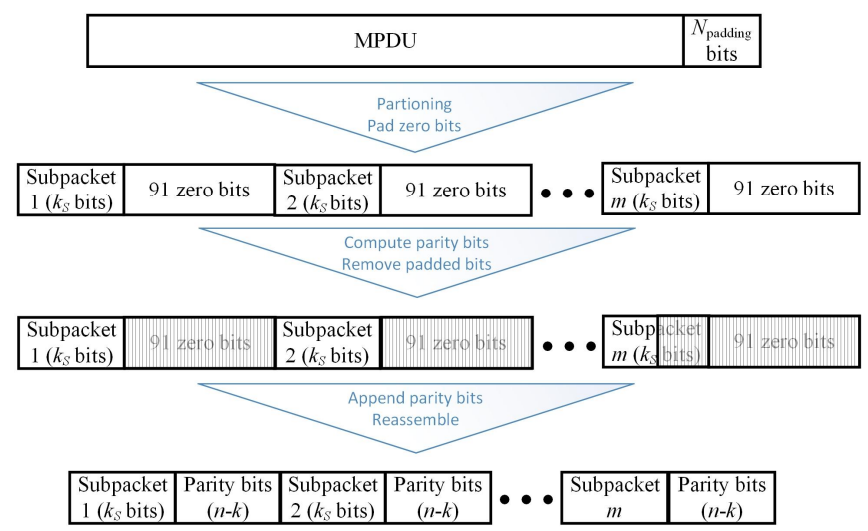

Fig. 4. Shortened BCH encoding process.

\section{B. Interference model}

The used interference model is based on the measurement campaigns at the Oulu university hospital. The campaigns were carried out to collect experimental data on channel usage at the $2.35-2.50 \mathrm{GHz}$ frequency band. By using the channel occupancy data, a mathematical model of the interference was formulated for the frequency band SmartBAN operates. The detailed insight to the measurement campaigns and data analysis procedures are given in [7]-[10]. Table 1 gives the parameters 
applied in two measurement campaigns carried out in a daily surgery ward (DS) and accident \& emergency ward (AEW).

TABLE 1

PARAMETER SETTINGS FOR CAMPAIGNS

\begin{tabular}{lll}
\multicolumn{1}{c}{ Parameter } & \multicolumn{1}{c}{$\begin{array}{c}\text { DS } \\
\text { Value }\end{array}$} & \multicolumn{1}{c}{$\begin{array}{c}\text { AEW } \\
\text { Value }\end{array}$} \\
\hline Frequency band & $2.35 \mathrm{to} 2.50 \mathrm{GHz}$ & 2.35 to $2.50 \mathrm{GHz}$ \\
Bandwidth & $150 \mathrm{MHz}$ & $150 \mathrm{MHz}$ \\
Number of recorded & 1601 & 1200 \\
$c$ Resolution bandwidth & $300 \mathrm{kHz}$ & $250 \mathrm{kHz}$ \\
Bin-width & $93.7 \mathrm{kHz}$ & $125 \mathrm{kHz}$ \\
No. of sweeps & 10000 & $1250 \mathrm{per}$ minute \\
Sweep time & $2 \mathrm{~ms}$ approx. & $3 \mathrm{~ms}$ approx. \\
Processing time & $22 \mathrm{~ms}$ & $45 \mathrm{~ms}$ \\
Integration time & $4 \mathrm{minutes}$ & $1 \mathrm{minute}$ \\
Measurement duration & 7 days $(6 \times 24$ & 1 week \\
& hrs $+1 * 10 \mathrm{hrs})$ & \\
\hline \hline
\end{tabular}

In particular, three different channels have been evaluated: low, moderate and high interference among all the measurements. Channel $6(2437 \mathrm{MHz})$ of the measurement campaign in the DS is the best one (low interference scenario), Channel 1 $(2412 \mathrm{MHz})$ of the AEW measurement campaign is the worst one (high interference scenario), while Channel 6 of the AEW measurement campaign represents a moderate interference scenario. High interference channel showed a channel occupancy between $8 \%$ and $60 \%$ over one week. Moderate interference showed a channel occupancy between $4 \%$ and $7 \%$ over one week, while the low interference channel showed a channel occupancy in the range $0-4 \%$ over one week of measurements. Based on the measurements, the statistical model of the interference was extracted. Fig. 5 shows a comparison between the simulated interference instances for each scenario.

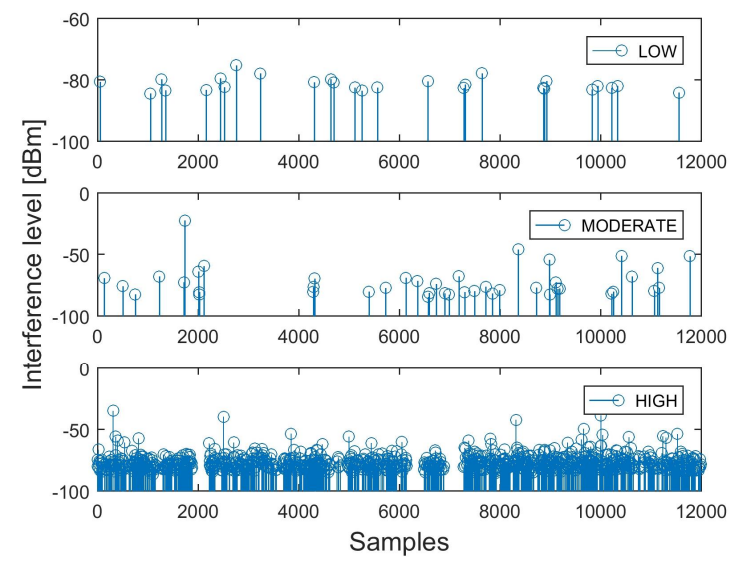

Fig. 5. Generated realizations for interference scenarios.

\section{Simulations}

Comprehensive set of simulations were carried out to study performance difference between two FEC schemes in the interfered IEEE 802.15.6 CM3 channel. The studied coding schemes were $\mathrm{BCH}(127,113)$ and $\mathrm{BCH}(36,22)$ using the 1-, 2- or 4-time
PPDU repetition $\left(N_{\text {PPDU }}\right)$ as discussed above. Table 2 summarizes the simulation parameters.

TABLE 2

SIMULATION PARAMETERS

\begin{tabular}{lc}
\hline \multicolumn{1}{c}{ Parameter } & Value(s) \\
\hline PPDU repetition $\left(N_{\text {PPDU }}\right)$ & $1,2,4$ \\
BCH code rate & $(127,113),(36,22)$ \\
MAC frame body [octets] & 50 \\
Samples per GFSK symbol & 20 \\
Pulse length of GFSK [symbols] & 1 \\
Traceback depth of GFSK demodulator & 10 \\
Distance [cm] & 45 \\
Interference scenario & low, high \\
Number of interference realizations & 100 \\
\hline \hline
\end{tabular}

\section{A. Results}

Fig. 6 - Fig. 10 show the simulation results for both code rates and the PPDU repetition of 1, 2 or 4 with several signal-tointerference power ratio (SIR) values in the low and high interference scenarios. The results show that SmartBAN system using the $\mathrm{BCH}$ coding with higher coding rate performs better in an interfered channel, as was expected. The shortened version of the $\mathrm{BCH}(127,113)$ code uses shorter message words but the same number of parity bits as its primitive code, which explains the performance improvement. In the low interference scenario, difference between coding schemes is not remarkable. But when the presence of interference increases, the difference in performance arises. The shortened $\mathrm{BCH}$ can gain the frame error rate (FER) level of $1 \%$ when SIR is more than $6 \mathrm{~dB}$ and $N_{\text {PPDU }}=1$, whereas the primitive code cannot attain it even with $\mathrm{SIR}=9$ $\mathrm{dB}$. Using the 2-time repetition, the performance rises overall for both coding rates. When the repetition is 4 , the shortened code can perform well when SIR is more than $3 \mathrm{~dB}$ in the high interference scenario. The results show that using the better encoding for PPDU than specified in the SmartBAN specification, the lower error level can be gained even in the high interference scenario. Note that there is no interference mitigation technique included in the SmartBAN standard yet and therefore no mitigation is utilized in the simulations either.

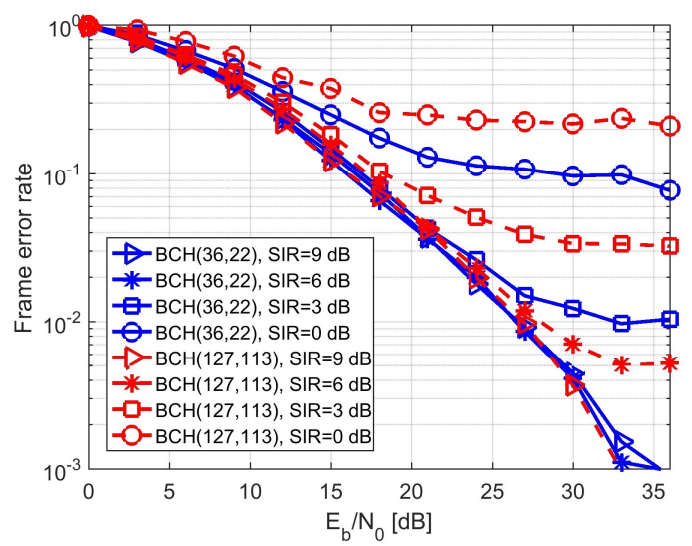

Fig. 6. Frame error rate in the low interference scenario, $N_{\mathrm{PPDU}}=1$. 


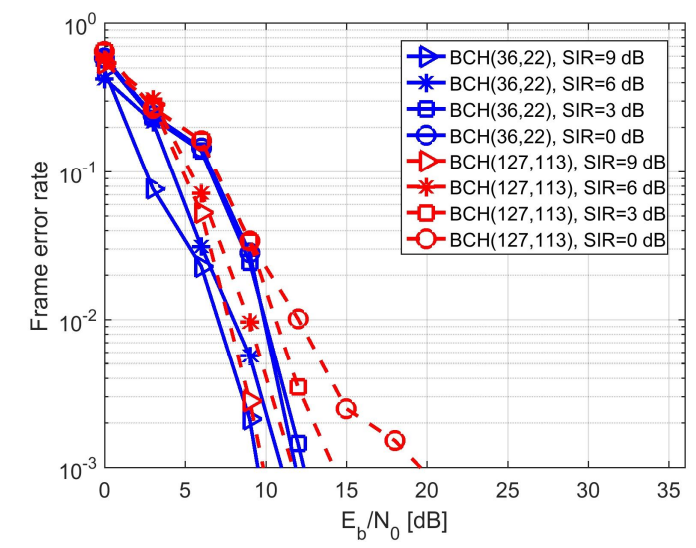

Fig. 7. Frame error rate in the low interference scenario, $N_{\text {PPDU }}=4$.

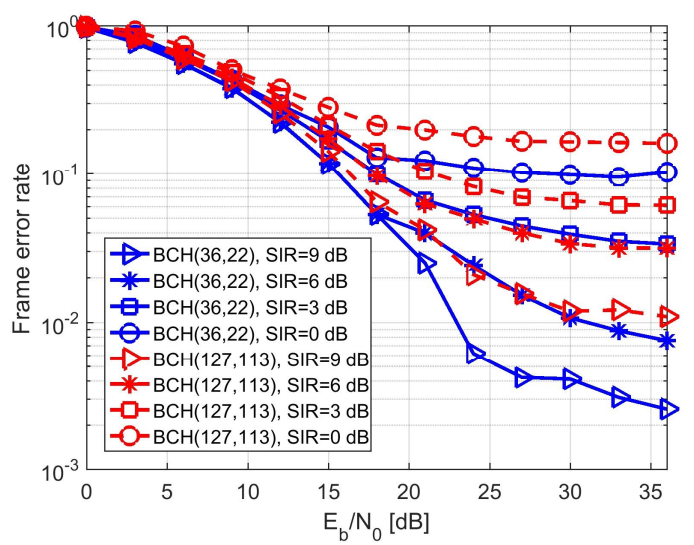

Fig. 8. Frame error rate in the high interference scenario, $N_{\text {PPDU }}=1$.

\section{CONCLUSION}

In this paper, the $\mathrm{BCH}(127,113)$ and shortened $(36,22)$ encoding schemes for the SmartBAN system were studied in the interfered IEEE 802.15.6 CM3 channel model. The interference model utilized was based on the measurement campaigns at the Oulu University Hospital, Finland.

The $\mathrm{BCH}(36,22)$ is shortened version from the primitive $\mathrm{BCH}(127,113)$. The primitive code is used for error control of MPDU, whereas the shortened version encodes the PLCP header. In this study, we applied this encoding also for PPDU. It uses the same number of the parity bits as its primitive code but shorter message word. The simulation results showed that the performance improvement can be gained by using the shortened version of the primitive $\mathrm{BCH}$ code in the interfered on-body to on-body channel. By using the shortened version $\mathrm{BCH}$ code, the lower error level can be gained even in the high interference scenario.

\section{REFERENCES}

[1] Smart Body Area Network (SmartBAN); Enhanced Ultra-Low Power Physical Layer, ETSI TS 103326 V1.1.1, April, 2015.

[2] Smart Body Area Network (SmartBAN); Low Complexity Medium Access Control (MAC) for SmartBAN, ETSI TS 103325 V1.1.1, April, 2015.

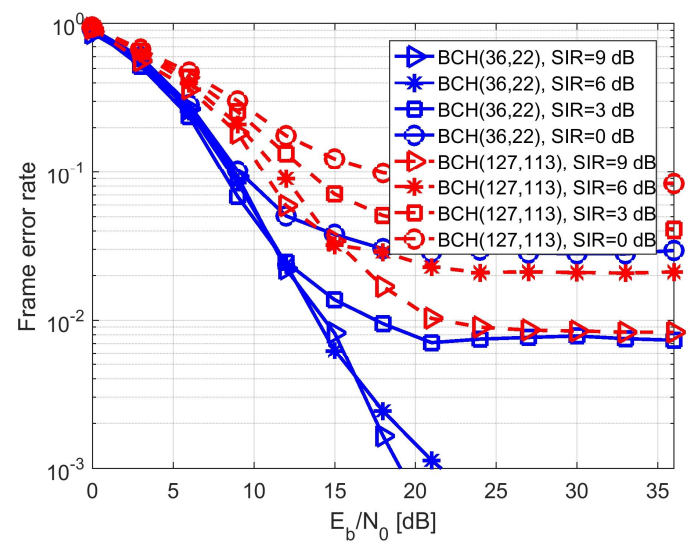

Fig. 9. Frame error rate in the high interference scenario, $N_{\text {PPDU }}=2$.

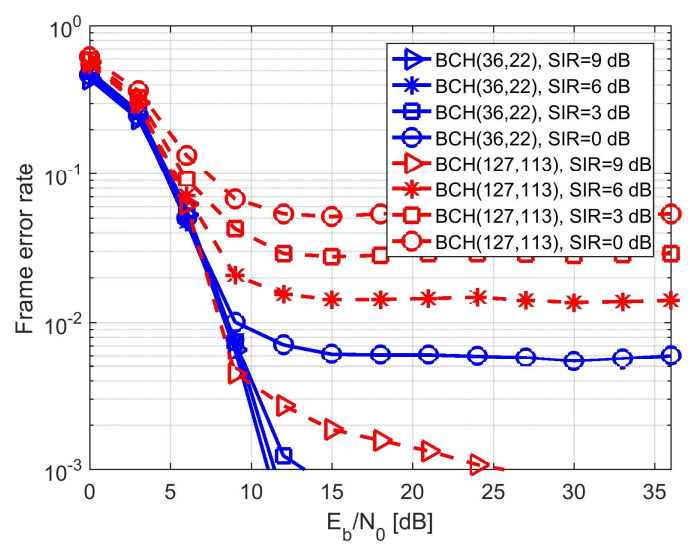

Fig. 10. Frame error rate in the high interference scenario, $N_{\mathrm{PPDU}}=4$

[3] Smart Body Area Networks (SmartBAN); Unified data representation formats, semantic and open data model, ETSI TS 103378 V1.1.1, December 2015.

[4] Smart Body Area Networks (SmartBAN): System Level Description and Requirements", ETSI TR 103 394, early draft V0.9.2 (2016-10), unpublished.

[5] Smart Body Area Networks (SmartBAN): Measurement and Modelling of SmartBAN RF Environment, ETSI TR 103 395, unpublished.

[6] K.Y. Yazdandoost and K. Sayrafian-Pour, "Channel Model for Body Area Network (BAN),” IEEE P802.15 Wireless Personal Area Networks, Tech. Rep. IEEE P802.15-08-780-09-0006, April, 2009.

[7] J. Lehtomäki, R. Vuohtoniemi, K. Umebayashi and J-P. Mäkelä, "Energy Detection Based Estimation of Channel Occupancy Rate With Adaptive Noise Estimation," IEICE Transactions on Communications, E95B: 04, Page(s):1076-1084, April, 2012

[8] M. H. Virk, R. Vuohtoniemi, M. Hämäläinen, J.-P. Mäkelä and J. Iinatti, "Spectrum Occupancy Evaluations at 2.35-2.50 GHz ISM Band in a Hospital Environment, " in Proc. BodyNets-2014, London, UK, 2014.

[9] L. Mucchi, A. Carpini, T. D’Anna, M. H. Virk, M. Hamalainen and J. Iinatti, "Threshold Setting for the Evaluation of the Aggregate Interference in ISM Band in Hospital Environments," in Proc. ISMICT2015, Kamakura, Japan, 2015.

[10] M. H. Virk, R. Vuohtoniemi, M. Hämäläinen, J. Iinatti and J-P. Mäkelä, "Stochastic Spectral Occupancy Modeling: A Body Area Network Perspective in ISM Band," in Proc. ISMICT2015, Kamakura, Japan, 2015 